\title{
Explicit formulae for Kerov polynomials
}

\author{
P. Petrullo $\cdot$ D. Senato
}

Received: 4 September 2009 / Accepted: 27 May 2010 / Published online: 16 June 2010

(C) Springer Science+Business Media, LLC 2010

\begin{abstract}
We prove two formulae expressing the Kerov polynomial $\Sigma_{k}$ as a weighted sum over the set of noncrossing partitions of the set $\{1, \ldots, k+1\}$. We also give a combinatorial description of a family of symmetric functions specializing in the coefficients of $\Sigma_{k}$.
\end{abstract}

Keywords Kerov polynomials · Noncrossing partitions $\cdot$ Symmetric group Normalized characters $\cdot$ Symmetric functions

\section{Introduction}

The $n$th free cumulant $R_{n}$ can be thought of as a function $R_{n}: \lambda \in \mathcal{Y} \mapsto R_{n}(\lambda) \in \mathbb{Z}$, defined on the set of all Young diagrams $\mathcal{Y}$, which we identify with the corresponding integer partitions, and taking integer values. Indeed, after a suitable representation of a Young diagram $\lambda$ as a function in the plane $\mathbb{R}^{2}$ [4], it is possible to determine the sequences of integers $x_{0}, \ldots, x_{m}$ and $y_{1}, \ldots, y_{m}$, consisting of the $x$-coordinates of the local minima and maxima of $\lambda$, respectively. In this way, if we set

$$
\mathcal{H}_{\lambda}(z)=\frac{\prod_{i=0}^{m}\left(z-x_{i}\right)}{\prod_{i=1}^{m}\left(z-y_{i}\right)},
$$

then $R_{n}(\lambda)$ is the coefficient of $z^{n-1}$ in the formal Laurent series expansion of $\mathcal{K}_{\lambda}(z)$ such that $\mathcal{K}_{\lambda}\left(\mathcal{H}_{\lambda}(z)\right)=\mathcal{H}_{\lambda}\left(\mathcal{K}_{\lambda}(z)\right)=z$. It can be shown that $R_{1}(\lambda)=0$ for all $\lambda$.

P. Petrullo · D. Senato $(\varangle)$

Dipartimento di Matematica e Informatica, Università degli Studi della Basilicata, via dell'Ateneo

Lucano 10, 85100 Potenza, Italy

e-mail: domenico.senato@unibas.it

P. Petrullo

e-mail: p.petrullo@gmail.com 
So, the $k$ th Kerov polynomial is a polynomial $\Sigma_{k}\left(R_{2}, \ldots, R_{k+1}\right)$ which satisfies the following identity,

$$
\Sigma_{k}\left(R_{2}(\lambda), \ldots, R_{k+1}(\lambda)\right)=(n)_{k} \frac{\chi^{\lambda}\left(k, 1^{n-k}\right)}{\chi^{\lambda}\left(1^{n}\right)},
$$

where $\chi^{\lambda}\left(k, 1^{n-k}\right)$ denotes the value of the irreducible character of the symmetric group $\mathfrak{S}_{n}$ indexed by the partition $\lambda$ on $k$-cycles. Two remarkable properties of $\Sigma_{k}$ have to be stressed. First, it is a "universal polynomial", that is, it depends neither on $\lambda$ nor on $n$. Second, its coefficients are nonnegative integers. A combinatorial proof of the positivity of $\Sigma_{k}$ is quite recent and is due to Féray [8]. An explicit combinatorial description of such coefficients is due to Dołęga, Féray and Śniady [6]. Until now, several results on Kerov polynomials have been proved and conjectured; see, for instance, [5, 10, 12, 17] and [9] for a more detailed treatment.

Originally, free cumulants arise in the noncommutative context of free probability theory [15]. To the best of our knowledge, their earliest applications in the asymptotic character theory of the symmetric group are due to Biane; see, for instance, [3]. In 1994, Speicher [16] showed that the formulae connecting moments and free cumulants of a noncommutative random variable $X$ obey the Möbius inversion on the lattice of noncrossing partitions of a finite set. This result highlights the strong analogy between free cumulants and classical cumulants, which are related to the moments of a random variable $X$, defined on a classical probability space, via the Möbius inversion on the lattice of all partitions of a finite set. More recently, Di Nardo, Petrullo and Senato [7] have shown how the classical umbral calculus provides an alternative setting for the cumulant families which passes through a generalization of the Abel polynomials.

In 1997, it was again Biane [2] who showed that the lattice $N C_{n}$ of noncrossing partitions of $\{1, \ldots, n\}$ can be embedded into the Cayley graph of the symmetric group $\mathfrak{S}_{n}$. Thus it seems reasonable that a not too complicated expression of the Kerov polynomials involving noncrossing partitions, or the Cayley graph of $\mathfrak{S}_{n}$, should exist. In particular, such a formula, conjectured in [4], appeared with a rather implicit description in $[6,8]$.

In this paper, we state two explicit formulae relating $\Sigma_{k}$ and the set $N C_{k+1}$ of noncrossing partitions of $\{1, \ldots, k+1\}$. More precisely, if $N C_{k+1}^{\text {irr }}$ denotes the subset of $N C_{k+1}$ of partitions having 1 and $k+1$ in the same block, then a new partial order $\leq{ }^{\mathrm{irr}}$ on $N C_{k+1}^{\mathrm{irr}}$ is considered, thanks to which we have

$$
\Sigma_{k}=\sum_{\tau \in N C_{k+1}^{\mathrm{irr}}}\left[\sum_{\tau \leq \mathrm{irr} \pi}(-1)^{\ell_{\pi}-1} W_{\tau}(\pi)\right] R_{\dot{\tau}} .
$$

Here, $\ell_{\pi}$ is the number of blocks of $\pi, W_{\tau}(\pi)$ is a suitable weight depending on $\tau$ and $\pi$, and $R_{i}=\prod_{B} R_{|B|}$, with $B$ ranging over the blocks of $\tau$ having at least 2 elements. The special structure of the weight $W_{\tau}(\pi)$ allows us to give a combinatorial description of the symmetric functions $g_{\mu}\left(x_{1}, \ldots, x_{k}\right)$ 's, that evaluated at $x_{i}=i$ return the coefficient of $\prod_{i \geq 2} R_{i}^{m_{i}}$ in $\Sigma_{k}$, for every integer partition $\mu$ of size $k+1$ having $m_{i}$ parts equal to $i$. 
A second formula expressing $\Sigma_{k}$ as a weighted sum over the whole $N C_{k+1}$ is proved by means of the notion of irreducible components of a noncrossing partition. In particular, if $d_{\tau}$ is the number of irreducible components of $\tau$, then we have

$$
\Sigma_{k}=\sum_{\tau \in N C_{k+1}}\left[(-1)^{d_{\tau}-1} V_{\tau}\right] R_{\dot{\tau}},
$$

where $V_{\tau}$ is a suitable weight depending on $\tau$.

\section{Kerov polynomials}

Let $n$ be a positive integer and let $\lambda=\left(\lambda_{1}, \ldots, \lambda_{l}\right)$ be an integer partition of size $n$, that is, $1 \leq \lambda_{1} \leq \cdots \leq \lambda_{l}$ and $\sum_{i} \lambda_{i}=n$. Denote by $\mathcal{Y}_{n}$ the set of all Young diagrams of size $n$, and set $\mathcal{Y}=\bigcup_{n} \mathcal{Y}_{n}$. From now on, an integer partition and its Young diagram will be denoted by the same symbol $\lambda$. Moreover, as is usual we write $\lambda \vdash n$ if $\lambda$ is an integer partition of size $n$.

After a suitable representation of a Young diagram $\lambda$ as a function in the plane $\mathbb{R}^{2}$ [4], it is possible to determine the sequences of integers $x_{0}, \ldots, x_{m}$ and $y_{1}, \ldots, y_{m}$, consisting of the $x$-coordinates of its local minima and maxima, respectively. Then, by expanding the rational function

$$
\mathcal{H}_{\lambda}(z)=\frac{\prod_{i=0}^{m}\left(z-x_{i}\right)}{\prod_{i=1}^{m}\left(z-y_{i}\right)}
$$

as a formal power series in $z^{-1}$ one has $\mathcal{H}_{\lambda}(z)=z^{-1}+\sum_{n \geq 1} M_{n}(\lambda) z^{-(n+1)}$. The integer $M_{n}(\lambda)$ is said to be the $n$th moment of $\lambda$. Now, define $\mathcal{K}_{\lambda}(z)=\mathcal{H}_{\lambda}^{\langle-1\rangle}(z)$, that is $\mathcal{K}_{\lambda}\left(\mathcal{H}_{\lambda}(z)\right)=\mathcal{H}_{\lambda}\left(\mathcal{K}_{\lambda}(z)\right)=z$, and consider its expansion as a formal Laurent series, $\mathcal{K}_{\lambda}(z)=z^{-1}+\sum_{n \geq 1} R_{n}(\lambda) z^{n-1}$. Then, the integer $R_{n}(\lambda)$ is named the $n$th free cumulant of $\lambda$. It is not difficult to see that $M_{1}(\lambda)=R_{1}(\lambda)=0$ for all $\lambda$. By setting $\mathcal{M}_{\lambda}(z)=z^{-1} \mathcal{H}_{\lambda}\left(z^{-1}\right)$ and $\mathcal{R}_{\lambda}(z)=z_{\lambda}(z)$, we obtain two formal power series in $z, \mathcal{M}_{\lambda}(z)=1+\sum_{n \geq 1} M_{n}(\lambda) z^{n}$ and $\mathcal{R}_{\lambda}(z)=1+\sum_{n \geq 1} R_{n}(\lambda) z^{n}$, such that

$$
\mathcal{M}_{\lambda}(z)=\mathcal{R}_{\lambda}\left(z \mathcal{M}_{\lambda}(z)\right)
$$

Let $\mu \vdash n$ and denote by $\chi^{\lambda}(\mu)$ the value of the irreducible character of $\mathfrak{S}_{n}$ indexed by $\lambda$ on the permutations of type $\mu$. So that, if $\mu=\left(k, 1^{n-k}\right)$, that is, $\mu_{1}=k$ and $\mu_{2}=\cdots=\mu_{n-k+1}=1$, then the value $\widehat{\chi}^{\lambda}\left(k, 1^{n-k}\right)$ of the normalized character indexed by $\lambda$ on the $k$-cycles of $\mathfrak{S}_{n}$ is given by

$$
\widehat{\chi}^{\lambda}\left(k, 1^{n-k}\right)=(n)_{k} \frac{\chi^{\lambda}\left(k, 1^{n-k}\right)}{\chi^{\lambda}\left(1^{n}\right)},
$$

where $(n)_{k}=n(n-1) \cdots(n-k+1)$. The $k$ th Kerov polynomial is a polynomial $\Sigma_{k}$ in $k$ commuting variables which satisfies the following interesting identity,

$$
\Sigma_{k}\left(R_{2}(\lambda), \ldots, R_{k+1}(\lambda)\right)=\widehat{\chi}^{\lambda}\left(k, 1^{n-k}\right) .
$$


If we think of $R_{n}(\lambda)$ as the image of a map $R_{n}: \lambda \in \mathcal{Y} \mapsto R_{n}(\lambda) \in \mathbb{Z}$, then also Kerov polynomials become maps $\Sigma_{k}=\Sigma_{k}\left(R_{2}, \ldots, R_{k+1}\right)$, which are polynomials in the $R_{n}$ 's, such that $\Sigma_{k}(\lambda)=\widehat{\chi}^{\lambda}\left(k, 1^{n-k}\right)$. Since the coefficients of $\Sigma_{k}$ depend neither on $\lambda$ nor on $n$, but only on $k$, such polynomials are said to be "universal". A second remarkable property of Kerov polynomials is that all their coefficients are positive integers. This fact is known as the "Kerov conjecture" [11]. The first proof of the Kerov conjecture was given with combinatorial methods by Féray [8]. By using rather different techniques, the same author with Dołega and Śniady [6] have then stated an explicit combinatorial interpretation of such coefficients. The following formula for $\Sigma_{k}$ can be found in Stanley [17]. It is also stated in Biane [4], where the author quotes it as a private communication with A. Okounkov.

Theorem 2.1 Let $\mathcal{R}(z)=1+\sum_{n \geq 2} R_{n} z^{n}$. If $\mathcal{F}(z)=\frac{z}{\mathcal{R}(z)}$ and $\mathcal{G}(z)=\frac{z}{\mathcal{F}^{\langle-1\rangle}\left(z^{-1}\right)}$, then we have

$$
\Sigma_{k}=-\frac{1}{k}\left[z^{-1}\right]_{\infty} \prod_{j=0}^{k-1} \mathcal{G}(z-j)
$$

More precisely, if $\left[z^{n}\right] f(z)$ denotes the coefficient of $z^{n}$ in the formal power series $f(z)$, then $\left[z^{-1}\right]_{\infty} f(z)=[z] f\left(z^{-1}\right)$. This way, Identity (2.2) states that $\Sigma_{k}$ is obtained by expressing the right-hand side in terms of the free cumulants $R_{n}$ 's. Moreover, thanks to the invariance of the residue under translation of the variable, if $\mathcal{M}(z)=1+\sum_{n \geq 1} M_{n} z^{n}$, then by virtue of (2.1) we have $z \mathcal{G}(z)^{-1}=\mathcal{M}\left(z^{-1}\right)$, and (2.2) can be rewritten in the following equivalent form,

$$
\Sigma_{k}=-\frac{1}{k}\left[z^{k+1}\right] \prod_{j=1}^{k} \frac{1-j z}{\mathcal{M}\left(\frac{z}{1-j z}\right)}
$$

For all $j=1, \ldots, k$, we denote by $\lambda \boxplus j$ the image of the diagram $\lambda$ under the translation of the plane given by $x \mapsto x+j$. The $i$ th local minimum and maximum of $\lambda \boxplus j$ are $x_{i}+j$ and $y_{i}+j$, respectively, so that

$$
\mathcal{H}_{\lambda \boxplus j}(z)=\frac{\prod_{i=0}^{m} z-\left(x_{i}+j\right)}{\prod_{i=1}^{m} z-\left(y_{i}+j\right)} \quad \text { and } \quad \mathcal{M}_{\lambda \boxplus j}(z)=\frac{1}{1-j z} \mathcal{M}_{\lambda}\left(\frac{z}{1-j z}\right) .
$$

In this way, we may rewrite (2.3) as follows:

$$
\Sigma_{k}=-\frac{1}{k}\left[z^{k+1}\right] \prod_{j=1}^{k} \frac{1}{\mathcal{M}_{\lambda \boxplus j}(z)}
$$

Denote by $\mathcal{R}_{\lambda \boxplus j}(z)$ the formal power series such that $\mathcal{M}_{\lambda \boxplus j}(z)=\mathcal{R}_{\lambda \boxplus j}\left(z \mathcal{M}_{\lambda \boxplus j}(z)\right)$. It is immediate to verify that

$$
\mathcal{R}_{\lambda \boxplus j}(z)=j z+\mathcal{R}_{\lambda}(z)
$$




\section{Irreducible noncrossing partitions}

A partition of a finite set $S$ is an unordered sequence $\tau=\left\{A_{1}, \ldots, A_{l}\right\}$ of pairwise disjoint nonempty subsets of $S$, called the blocks of $\tau$, such that $\bigcup_{i} A_{i}=S$. We say that $\tau$ refines $\pi$, written $\tau \leq \pi$, if and only if each block of $\pi$ is a union of blocks of $\tau$. If $T \subset S$, then the restriction of $\tau$ to $T$ is the partition $\tau_{\left.\right|_{T}}$ obtained by removing from $\tau$ all the elements in $S \backslash T$.

Denote by $[n]$ the set $\{1, \ldots, n\}$. A partition $\tau=\left\{A_{1}, \ldots, A_{l}\right\}$ of $[n]$ is said to be noncrossing if and only if $a, c \in A_{i}$ and $b, d \in A_{j}$ implies $i=j$, whenever $1 \leq$ $a<b<c<d \leq n$. The set of all the noncrossing partitions of [ $n]$ is usually denoted by $N C_{n}$. Its cardinality equals the $n$th Catalan number $C_{n}=\frac{1}{n+1}\left(\begin{array}{c}2 n \\ n\end{array}\right)$. The reader interested in this subject may refer to [1] and references therein for further details. Now, if we set $R_{\tau}=R_{\left|A_{1}\right|} \cdots R_{\left|A_{l}\right|}$ then we can state the following beautiful formula, due to Speicher [16], expressing the moments $M_{n}$ 's in terms of their respective free cumulants $R_{n}$ 's:

$$
M_{n}=\sum_{\tau \in N C_{n}} R_{\tau}
$$

Following Lehner [13], if 1 and $n$ lie in the same block of a partition $\tau$ of [ $n$ ], then we say that $\tau$ is irreducible. Moreover, we denote by $N C_{n}^{\text {irr }}$ the set of all the irreducible noncrossing partitions of $[n]$. Note that a partition of $N C_{n+1}^{\text {irr }}$ is obtained from a partition of $N C_{n}$ simply by inserting $n+1$ in the block containing 1 . By taking the sum of the monomials $R_{\tau}$ 's, $\tau$ ranging in $N C_{n}^{\text {irr }}$ instead of $N C_{n}$, one defines a quantity $B_{n}$ known as a boolean cumulant (see [13])

$$
B_{n}=\sum_{\tau \in N C_{n}^{\mathrm{irr}}} R_{\tau}
$$

In particular, if $\mathcal{B}(z)=\sum_{n \geq 1} B_{n} z^{n}$, then we have

$$
\mathcal{M}(z)=\frac{1}{1-\mathcal{B}(z)}
$$

If $\mu=\left(\mu_{1}, \ldots, \mu_{l}\right)$ is an integer partition of size $n$, set $R_{\mu}=R_{\mu_{1}} \cdots R_{\mu_{l}}$ and define $N C_{\mu}^{\text {irr }}$ to be the subset of $N C_{n}^{\text {irr }}$ consisting of all the irreducible noncrossing partitions of type $\mu$. From (3.1) we have

$$
B_{n}=\sum_{\mu \vdash n}\left|N C_{\mu}^{\mathrm{irr}}\right| R_{\mu}
$$

Moreover, thanks to the Lagrange inversion formula, we recover

$$
B_{n}=\frac{1}{n-1}\left[z^{n}\right] \mathcal{R}(z)^{n-1}=\sum_{\mu \vdash n} \frac{(n-2) \ell_{\mu}-1}{m(\mu) !} R_{\mu},
$$


where $m(\mu) !=m_{1}(\mu) ! \cdots m_{n}(\mu) !$, and $m_{i}(\mu)$ is the number of occurrences of $i$ as a part of $\mu$. By comparing (3.3) and (3.4), we deduce

$$
\left|N C_{\mu}^{\mathrm{irr}}\right|=\frac{(n-2)_{\ell_{\mu}-1}}{m(\mu) !}
$$

The notion of noncrossing partition can be given for any totally ordered set $S$. In particular, $N C_{S}^{\text {irr }}$ will denote the set of all the noncrossing partitions of $S$, such that the minimum and the maximum of $S$ lie in the same block. Let us introduce a partial order on $N C_{S}^{\text {irr }}$.

Definition 3.1 (Irreducible refinement) Let $\tau, \pi \in N C_{S}^{\mathrm{irr}}$. We say that $\tau$ refines $\pi$ in an irreducible way, and write $\tau \leq{ }^{\text {irr }} \pi$, if and only if $\tau \leq \pi$ and the restriction $\pi_{\left.\right|_{A}}$, of $\pi$ to each block $A$ of $\tau$, is in $N C_{A}^{\text {irr }}$. In particular, we say that $\pi \operatorname{covers} \tau$ if and only if $\tau \leq \leq^{\mathrm{irr}} \pi$ and $\pi$ is obtained by joining two blocks of $\tau$.

For instance, let $\tau=\{\{1,5\},\{2,3\},\{4\}\}, \pi=\{\{1,2,3,5\},\{4\}\}$ and $\sigma=\{\{1,5\}$, $\{2,3,4\}\}$. Then $\tau, \pi, \sigma \in N C_{5}^{\text {irr }}$ and $\tau$ refines both $\pi$ and $\sigma$. However, $\tau \leq{ }^{\text {irr }} \pi$ and in particular $\pi$ covers $\tau$, while it is not true that $\tau \leq{ }^{\text {irr }} \sigma$, since $\tau_{\mid\{2,3,4\}}=\{\{2,3\},\{4\}\}$ is not irreducible.

The singletons of the noncrossing partitions will play a special role. For all $\tau \in$ $N C_{n}$, we denote by $U(\tau)$ the subset of $[n]$ consisting of all the integers $i$ such that $\{i\}$ is a block of $\tau$, while $\dot{\tau}$ will be the partition obtained from $\tau$ by removing its singletons. When $\tau, \pi \in N C_{n}^{\text {irr }}$ and $\tau \leq^{\text {irr }} \pi$, then $\pi_{\tau}$ is the restriction of $\pi$ to $U(\tau)$. Note that $\pi_{\tau} \in N C_{U(\tau)}$.

We define a tree-representation for the partitions of $N C_{n}^{\text {irr }}$ in the following way. Assume $\tau=\left\{A_{1}, \ldots, A_{l}\right\} \in N C_{n}^{\text {irr }}$ and $\min A_{i}<\min A_{i+1}$. Construct a labeled rooted tree $t_{\tau}$ by the following steps:

- Choose $A_{1}$ as the root of $t_{\tau}$;

- If $2 \leq i<j \leq l$ then draw an edge between $A_{i}$ and $A_{j}$ if and only if $i$ is the biggest integer such that $\min A_{i}<\min A_{j} \leq \max A_{j}<\max A_{i}$;

- Label each edge $\left\{A_{i}, A_{j}\right\}$ with $\min A_{j}$.

For example, if $\tau=\{\{1,2,7,12\},\{3,5,6\},\{4\},\{8,9\},\{10,11\}\}$ then $t_{\tau}$ is the following tree,
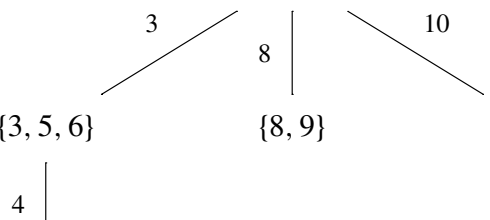

$\{4\}$

Now, let $E(\tau)$ be the set of labels of $t_{\tau}$, and choose $j \in E(\tau)$. We denote by $t_{\tau, j}$ the tree obtained from $t_{\tau}$ by deleting the edge labeled by $j$ and joining its nodes (i.e., 
joining the blocks). In the following, we will say that $t_{\tau, j}$ is the tree obtained from $t_{\tau}$ by removing $j$. Hence, $t_{\tau, 3}$ is given by

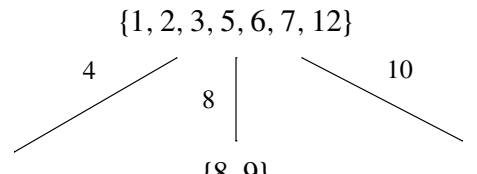

$\{8,9\}$

$\{10,11\}$

Now, $t_{\tau, j}$ is the tree-representation of an irreducible noncrossing partition, here denoted by $\tau_{\{j\}}$, whose blocks are the nodes of $t_{\tau, j}$. By construction, we have $\tau \leq{ }^{\text {irr }} \tau_{\{j\}}$ and $E\left(\tau_{\{j\}}\right)=E(\tau) \backslash\{j\}$. More generally, given a subset $S \subseteq E(\tau)$, we denote by $\tau_{S}$ the only partition whose tree $t_{\tau_{S}}$ is obtained from $t_{\tau}$ by successively removing all labels in $S$. We note that $\tau_{\emptyset}=\tau$ and that $\tau_{S}$ depends only on the set $S$ and not on the order in which labels are chosen. The following proposition is easy to prove.

Proposition 3.1 Let $\tau, \pi \in N C_{n}^{\mathrm{irr}}$. Then, we have $\tau \leq^{\mathrm{irr}} \pi$ if and only if $\pi=\tau_{S}$ for some $S \subseteq E(\tau)$. In particular, if $\ell(\tau)$ is the number of blocks of $\tau$, then we have

$$
\left|\left\{\sigma \mid \tau \leq^{\mathrm{irr}} \sigma\right\}\right|=\left|2^{E(\tau)}\right|=2^{\ell(\tau)-1},
$$

where $2^{E(\tau)}$ is the powerset of $E(\tau)$, and

$$
|\{\sigma \mid \sigma \operatorname{covers} \tau\}|=|E(\tau)|=\ell(\tau)-1 \text {. }
$$

\section{Kerov polynomial formulae}

By means of the results of previous sections, we are able to give new formulae for the Kerov polynomials $\Sigma_{k}$. In particular, the first formula is related to the partial order $\leq{ }^{\text {irr }}$ on the irreducible noncrossing partitions of the set $[k+1]$, instead the second formula expresses $\Sigma_{k}$ as a weighted sum over $N C_{k+1}$.

4.1 Kerov polynomials and irreducible refinement

Let $\tau, \pi \in N C_{k+1}^{\text {irr }}$ with $\tau \leq^{\mathrm{irr}} \pi$, and let $\pi_{\tau}=\left\{A_{1}, \ldots, A_{l}\right\}$. Moreover, set $A_{i}=\emptyset$ for $i>l$ and define $W_{\tau}(\pi)$ to be such that

$$
W_{\tau}(\pi)=\frac{1}{k !} \sum_{w \in \mathfrak{S}_{k}} w(1)^{\left|A_{1}\right|} \cdots w(k)^{\left|A_{k}\right|} .
$$

Theorem 4.1 We have

$$
\Sigma_{k}=\sum_{\tau \in N C_{k+1}^{\mathrm{irr}}}\left[\sum_{\tau \leq \mathrm{irr} \pi}(-1)^{\ell_{\pi}-1} W_{\tau}(\pi)\right] R_{\dot{\tau}} .
$$


Proof Let $B_{n}(j)=-\left[z^{n}\right]\left(\mathcal{M}_{\lambda \boxplus j}(z)\right)^{-1}$ for all $n \geq 1$, and set $B_{0}(j)=1$. Since $R_{1}(\lambda)=0$, then from (2.5), (3.1) and (3.2) we deduce

$$
B_{n}(j)=\sum_{\pi \in N C_{n}^{\mathrm{irr}}} j^{u(\pi)} R_{\dot{\pi}}(\lambda),
$$

where $u(\pi)=|U(\pi)|$. The right-hand side in (2.4) is equal to

$$
-\frac{1}{k} \sum_{\substack{a_{1}, \ldots, a_{k} \geq 0 \\ a_{1}+\cdots+a_{k}=k+1}} \prod_{j=1}^{k}\left[z^{a_{j}}\right] \frac{1}{M_{\lambda \boxplus j}(z)}=\frac{1}{k} \sum_{\substack{\mu \vdash k+1 \\ \ell_{\mu} \leq k}} \frac{(-1)^{\ell_{\mu}-1}}{m(\mu) !\left(k-\ell_{\mu}\right) !} \sum_{w \in \mathfrak{S}_{k}} \prod_{i=1}^{k} B_{\mu_{i}}(w(i)),
$$

where $\mu_{1}, \ldots, \mu_{\ell_{\mu}}$ are the parts of $\mu$ and $\mu_{i}=0$ when $i>\ell_{\mu}$. However, by taking into account (3.5), we may rewrite it in the following form:

$$
\frac{1}{k !} \sum_{\pi \in N C_{k+1}^{\mathrm{irr}}}(-1)^{\ell_{\pi}-1} \sum_{w \in \mathfrak{S}_{k}} \prod_{i=1}^{k} B_{\left|A_{i}\right|}(w(i))
$$

where $A_{1}, \ldots, A_{\ell_{\mu}}$ are the blocks of $\pi$ and $A_{i}=\emptyset$ if $i>\ell_{\pi}$. Moreover, by means of identity (4.2), we obtain

$$
\sum_{w \in \mathfrak{S}_{k}} \prod_{i=1}^{k} B_{\left|A_{i}\right|}(w(i))=\sum_{\tau_{1}, \ldots, \tau_{k}} \sum_{w \in \mathfrak{S}_{k}} w(1)^{u\left(\tau_{1}\right)} \cdots w(k)^{u\left(\tau_{k}\right)} R_{\dot{\tau}_{1}}(\lambda) \cdots R_{\dot{\tau}_{k}}(\lambda),
$$

where $\tau_{i}$ ranges over all $N C_{A_{i}}^{\text {irr }}$, with $N C_{\emptyset}^{\text {irr }}=\{\emptyset\}$.

Now, if we set $\tau=\tau_{1} \cup \cdots \cup \tau_{k}$, then $\tau \in N C_{k+1}^{\mathrm{irr}}, \tau \leq \leq^{\mathrm{irr}} \pi$ and $R_{\dot{\tau}}(\lambda)=$ $R_{\dot{\tau}_{1}}(\lambda) \cdots R_{\dot{\tau}_{k}}(\lambda)$. Finally, $u\left(\tau_{i}\right)$ is the number of singletons in $\tau_{i}=\tau_{\left.\right|_{A_{i}}}$, that is, the cardinality of the set $A_{i} \cap U(\tau)$, which if nonempty is a block of $\pi_{\tau}$. This completes the proof.

Remark 4.1 Consider the polynomial $\Omega_{k}\left(x_{1}, \ldots, x_{k}\right)$ defined by

$$
\Omega_{k}\left(x_{1}, \ldots, x_{k}\right)=-\frac{1}{k}\left[z^{k+1}\right] \prod_{j=1}^{k} \frac{1-x_{j} z}{\mathcal{M}\left(\frac{z}{1-x_{j} z}\right)} .
$$

Of course, $\Omega_{k}$ is symmetric with respect to the $x_{i}$ 's. Moreover, by virtue of (2.3), we obtain $\Omega_{k}(1,2, \ldots, k)=\Sigma_{k}$. A formula for $\Omega_{k}\left(x_{1}, \ldots, x_{k}\right)$ is obtained from (4.1) simply by replacing $w(j)$ with $x_{w(j)}$ in $W_{\tau}(\pi)$. More precisely, via Proposition 3.1, if $\mu$ is an integer partition of size $k+1$ then the coefficient of $R_{\dot{\mu}}$ in $\Omega_{k}\left(x_{1} \ldots, x_{k}\right)$ is

$$
\boldsymbol{g}_{\mu}\left(x_{1}, \ldots, x_{k}\right)=\sum_{\tau \in N C_{\mu}^{\mathrm{irr}}} \sum_{S \subseteq E(\tau)}(-1)^{|E(\tau)|-|S|} W_{\tau}\left(S ; x_{1}, \ldots, x_{k}\right) \text {, }
$$

where $R_{\dot{\mu}}=\prod_{i \geq 2} R_{i}^{m_{i}(\mu)}$, and where $W_{\tau}\left(S ; x_{1}, \ldots, x_{k}\right)$ is obtained by replacing $w(j)$ with $x_{w(j)}$ in $W_{\tau}\left(\tau_{S}\right)$. Now, let $\lambda_{\tau}(S)$ denote the integer partition corresponding 
to the type of $\pi_{\tau}$, with $\pi=\tau_{S}$. Then, it is not difficult to see that

$$
k ! W_{\tau}\left(S ; x_{1}, \ldots, x_{k}\right)=m\left(\lambda_{\tau}(S)\right) !\left(k-\ell\left(\lambda_{\tau}(S)\right)\right) ! \boldsymbol{m}_{\lambda_{\tau}(S)}\left(x_{1}, \ldots, x_{k}\right),
$$

with $\boldsymbol{m}_{\lambda_{\tau}(S)}\left(x_{1}, \ldots, x_{k}\right)$ being the monomial symmetric function indexed by the partition $\lambda_{\tau}(S)$ [14]. This way, the coefficient of $R_{\dot{\mu}}$ in $\Omega_{k}\left(x_{1}, \ldots, x_{k}\right)$ is a symmetric function $\boldsymbol{g}_{\mu}\left(x_{1}, \ldots, x_{k}\right)$ of degree $m_{1}(\mu)$. Assume that

$$
\boldsymbol{g}_{\mu}\left(x_{1}, \ldots, x_{k}\right)=\sum_{\lambda} g_{\mu, \lambda} \boldsymbol{m}_{\lambda}\left(x_{1}, \ldots, x_{k}\right) .
$$

The left-hand side of (4.3) says that, for every $\lambda$ of size $m_{1}(\mu)$, we have

$$
g_{\mu, \lambda}=\frac{1}{k !} \sum_{\tau \in N C_{\mu}^{\mathrm{irr}}} \sum_{\substack{S \subseteq E(\tau) \\ \lambda \tau(S)=\lambda}}(-1)^{|E(\tau)|-|S|} m(\lambda) !\left(k-\ell_{\lambda}\right) !,
$$

thus we have provided a combinatorial formula for the $g_{\mu, \lambda}$ 's.

Finally, we stress that the coefficients in the expressions of $\boldsymbol{g}_{\mu}$ in terms of the classical basis, and Schur basis, are not positive integers. Indeed, we have

$$
\boldsymbol{g}_{(3,1,1,1)}=\frac{4}{5} \boldsymbol{m}_{(1,1,1)}-\frac{3}{5} \boldsymbol{m}_{(1,2)}+\frac{4}{5} \boldsymbol{m}_{(3)} .
$$

\subsection{Kerov polynomials via irreducible components of noncrossing partitions}

We will state a second formula expressing $\Sigma_{k}$ as a weighted sum over the whole $N C_{k+1}$. To this end, we introduce the notion of an irreducible component of a noncrossing partition.

Given $\tau \in N C_{n}$, let $j_{1}$ be the greatest integer lying in the same block as 1 . Set $\tau_{1}=\tau_{\mid\left[j_{1}\right]}$ so that $\tau_{1}$ is an irreducible noncrossing partition of [ $\left.j_{1}\right]$. Now, let $j_{2}$ be the greatest integer lying in the same block of $j_{1}+1$ and set $\tau_{2}=\tau_{\left.\right|_{\left[j_{1}+1, j_{2}\right]} \text {. }}$. By iterating this process, we determine the sequence of irreducible noncrossing partitions $\tau_{1}, \ldots, \tau_{d}$, which we name the irreducible components of $\tau$, such that $\tau=\tau_{1} \cup \cdots \cup \tau_{d}$. For all $\tau \in N C_{n}$, we denote by $d_{\tau}$ the number of its irreducible components. Note that $d_{\tau}=1$ if and only if $\tau$ is an irreducible noncrossing partition.

Theorem 4.2 We have

$$
\Sigma_{k}=\sum_{\tau \in N C_{k+1}}\left[(-1)^{d_{\tau}-1} V_{\tau}\right] R_{i}
$$

where

$$
V_{\tau}=\frac{1}{k} \sum_{1 \leq i_{1}<\cdots<i_{d} \leq k} i_{1}{ }^{u\left(\tau_{1}\right)} \cdots i_{d}^{u\left(\tau_{d}\right)}
$$

if $d=d_{\tau}$. 
Proof Let $\mathcal{B}_{\lambda \boxplus j}(z)=1\left(\mathcal{M}_{\lambda \boxplus j}(z)\right)^{-1}$. From (2.4) we obtain

$$
\begin{aligned}
\Sigma_{k} & =-\frac{1}{k}\left[z^{k+1}\right] \prod_{j=1}^{k}\left(1-B_{\lambda \boxplus j}(z)\right) \\
& =\sum_{d=1}^{k} \frac{(-1)^{d-1}}{k} \sum_{1 \leq i_{1}<\cdots<i_{d} \leq k}\left[z^{k+1}\right] \mathcal{B}_{\lambda \boxplus i_{1}}(z) \cdots \mathcal{B}_{\lambda \boxplus i_{d}}(z) .
\end{aligned}
$$

Of course, the complex $B_{n}(j)=\left[z^{n}\right] \mathcal{B}_{\lambda \boxplus j}(z)$ is the $n$-boolean cumulant of $\lambda \boxplus j$ and satisfies (4.2). This way we deduce

$$
\left[z^{k+1}\right] \mathcal{B}_{\lambda \boxplus i_{1}}(z) \cdots \mathcal{B}_{\lambda \boxplus i_{d}}(z)=\sum_{\substack{a_{1}, \ldots, a_{d} \geq 1 \\ a_{1}+\cdots+a_{d}=k+1}} \sum_{\pi_{1}, \ldots, \pi_{d}} i_{1}{ }^{u\left(\pi_{1}\right)} \cdots i_{d}{ }^{u\left(\pi_{d}\right)} R_{\pi_{1}} \cdots R_{\pi_{d}},
$$

where $\pi_{i}$ ranges over $N C_{a_{i}}^{\text {irr }}$. Let $a_{0}=0$ and consider the intervals $A_{i}=\left[a_{0}+\cdots+\right.$ $\left.a_{i-1}+1, a_{0}+\cdots+a_{i}\right]$ for $i=1, \ldots, k+1$. Each translation $h \in\left[1, a_{i}\right] \mapsto h+a_{0}+$ $\cdots+a_{i-1} \in A_{i}$ induces a bijection $\pi \in N C_{a_{i}}^{\mathrm{irr}} \mapsto \tau \in N C_{A_{i}}^{\mathrm{irr}}$. Hence, in the identity above we may replace each $\pi_{i}$ with the corresponding $\tau_{i}$ obtaining

$$
\left[z^{k+1}\right] \mathcal{B}_{\lambda \boxplus i_{1}}(z) \cdots \mathcal{B}_{\lambda \boxplus i_{d}}(z)=\sum_{\substack{a_{1}, \ldots, a_{d} \geq 1 \\ a_{1}+\cdots+a_{d}=k+1}} \sum_{\tau_{1}, \ldots, \tau_{d}} i_{1}{ }^{u\left(\tau_{1}\right)} \cdots i_{d}{ }^{u\left(\tau_{d}\right)} R_{\tau_{1}} \cdots R_{\tau_{d}} .
$$

Now, set $\tau=\tau_{1} \cup \cdots \cup \tau_{d}$ so that $\tau \in N C_{k+1}, \tau_{i}$ is the $i$ th irreducible component of $\tau$, and (4.4) follows.

Acknowledgement The authors thank the referees for their useful remarks and suggestions improving the technical quality of the paper.

\section{References}

1. Armstrong, D.: Generalized noncrossing partitions and combinatorics of Coxeter groups. Mem. Am. Math. Soc. 202, 949 (2009)

2. Biane, P.: Some properties of crossings and partitions. Discrete Math. 175, 41-53 (1997)

3. Biane, P.: Representations of symmetric groups and free probability. Adv. Math. 138(1), 126-181 (1998)

4. Biane, P.: Characters of the Symmetric Group and Free Cumulants. Lecture Notes in Math., vol. 1815, pp. 185-200. Springer, Berlin (2003)

5. Biane, P.: On the formula of Goulden and Rattan for Kerov Polynomials. Sémin. Lothar. Comb. 55 (2006)

6. Dołęga, M., Féray, V., Śniady, P.: Explicit combinatorial interpretation of Kerov character polynomials as number of permutation factorizations. Adv. Math. (2010). doi:10.1016/j.aim.2010.02.011

7. Di Nardo, E., Petrullo, P., Senato, D.: Cumulants and convolutions via Abel polynomials. Eur. J. Combin. (2010). doi:10.1016/j.ejc.2010.03.002

8. Féray, V.: Combinatorial interpretation and positivity of Kerov's character polynomials. J. Algebr. Comb. 29, 473-507 (2009)

9. Féray, V.: Fonctions sur l'ensemble des diagrammes de Young: caractères du groupe symétrique et polynômes de Kerov. Ph.D. thesis (2009). Available at http://feray.fr/valentin/Math/Feray_these.pdf 
10. Goulden, I.P., Rattan, A.: An explicit form for Kerov's character polynomials. Trans. Am. Math. Soc. 359, 3669-3685 (2007)

11. Kerov, S.V.: Talk at IHP Conference (2000)

12. Lassalle, M.: Two positive conjectures for Kerov polynomials. Adv. Appl. Math. 41, 407-422 (2008)

13. Lehner, F.: Free cumulants and enumeration of connected partitions. Eur. J. Comb. 22, 1025-1031 (2002)

14. Macdonald, I.G.: Symmetric Functions and Hall Polynomials, 2nd edn. Oxford University Press, London (1995)

15. Nica, A., Speicher, R.: Lectures on the Combinatorics of Free Probability. Cambridge University Press, Cambridge (2006)

16. Speicher, R.: Multiplicative functions on the lattice on nocrossing partitions and free convolution. Math. Ann. 298, 611-628 (1994)

17. Stanley, R.P.: Kerov's character polynomial and irreducible symmetric group characters of rectangular shape. Transparencies from a talk at CMS meeting, Quebec City (2002) 